Article

\title{
Engineered Mesenchymal Stem Cells Expressing Stromal Cell-derived Factor-1 Improve Erectile Dysfunction in Streptozotocin-Induced Diabetic Rats
}

\author{
Seung Hwan Jeon ${ }^{1,2,3}$, Guan Qun Zhu ${ }^{1,2} \oplus$, Woong Jin Bae ${ }^{1,2}$, Sae Woong Choi ${ }^{1}$, \\ Hyun Cheol Jeong ${ }^{1,2}$, Hyuk Jin Cho ${ }^{1}$, U-Syn Ha ${ }^{1}$, Sung-Hoo Hong ${ }^{1}$, Ji Youl Lee ${ }^{1}$, \\ Eun Bi Kwon ${ }^{1,2}$, Hyo-Jin Kim ${ }^{4}$, Soon Min Lee ${ }^{4}$, Hey-Yon Kim ${ }^{4}$ and Sae Woong Kim 1,2,3,* \\ 1 Department of Urology, College of Medicine, The Catholic University of Korea, Seoul 06591, Korea; \\ shwan52@naver.com (S.H.J.); xiaoguanqun1@gmail.com (G.Q.Z.); bwoong@catholic.ac.kr (W.J.B.); \\ lifeisa9ame@catholic.ac.kr (S.W.C.); koulich@naver.com (H.C.J.); hyukjincho0403@gmail.com (H.J.C.); \\ ushamd@catholic.ac.kr (U.-S.H.); toomey@catholic.ac.kr (S.-H.H.); uroljy@catholic.ac.kr (J.Y.L.); \\ eunbi9859@naver.com (E.B.K.) \\ 2 Catholic Integrative Medicine Research Institute, The Catholic University of Korea, Seoul 06591, Korea \\ 3 Department of Biomedicine \& Health Sciences, The Catholic University of Korea, Seoul 06591, Korea \\ 4 Department of Stem cell therapy, SL BIGEN, Seongnam 13488, Korea; hjkim@slbigen.com (H.-J.K.); \\ smlee@slbigen.com (S.M.L.); hykim@slbigen.com (H.-Y.K.) \\ * Correspondence: ksw1227@catholic.ac.kr; Tel.: +82-2-2258-6226; Fax: +82-2-599-7839
}

Received: 25 September 2018; Accepted: 21 November 2018; Published: 23 November 2018

\begin{abstract}
Effective therapies for erectile dysfunction (ED) associated with diabetes mellitus (DM) are needed. In this study, the effects of stromal cell-derived factor-1 (SDF-1)-expressing engineered mesenchymal stem cells (SDF-1 eMSCs) and the relevant mechanisms in the corpus cavernosum of a streptozotocin (STZ)-induced DM ED rat model were evaluated. In a randomized controlled trial, Sprague-Dawley (SD) rats $(n=48)$ were divided into four groups $(n=12 /$ group): Normal (control), DM ED (diabetes induced by STZ), DM ED + BM-MSC (treated with bone marrow [BM]-derived MSCs), and DM ED + SDF-1 eMSC (treated with SDF-1-expressing BM-MSCs). After four weeks, intracavernosal pressure (ICP), an indicator of erectile function, was $0.75 \pm 0.07$ in the normal group, $0.27 \pm 0.08$ in the DM ED group, $0.42 \pm 0.11$ in the DM ED + BM-MSC group, and $0.58 \pm 0.11$ in the DM ED + SDF-1 eMSC group. BM-MSCs, especially SDF-1 eMSCs, improved ED $(p<0.05)$. SDF-1 eMSC treatment improved the smooth muscle content in the corpus cavernosum $(p<0.05)$. As SDF-1 expression increased, ED recovery improved. In the SDF-1 eMSC group, levels of neuronal nitric oxide synthase (nNOS) and phosphorylated endothelial NOS (p-eNOS) were higher than those in other groups $(p<0.05)$. In addition, high stromal cell-derived factor-1 (SDF-1) expression was associated with increased vascular endothelial growth factor (VEGF) and basic fibroblast growth factor (bFGF) in DM ED rats $(p<0.05)$. Higher levels of phosphorylated protein kinase B (p-AKT)/protein kinase B (AKT) $(p<0.05)$ and B-cell lymphoma-2 (Bcl-2) and lower levels of the apoptosis factors Bcl2-associated $x$ (Bax) and caspase-3 were observed in the MSC-treated group than in the DM ED group $(p<0.05)$. SDF-1 eMSCs showed beneficial effects on recovery from erectile function.
\end{abstract}

Keywords: stromal cell-derived factor-1 expressing engineered mesenchymal stem cells; erectile dysfunction; intracavernosal injection; streptozotocin-induced diabetic rats

\section{Introduction}

Increased living standards have been accompanied by an increased demand for erectile dysfunction (ED) therapy, especially in elderly men who suffer from diabetes mellitus (DM) $[1,2]$. 
The main current treatment strategy for ED is phosphodiesterase 5 inhibitor (PDE5I), which affects angiectasis of the corpus cavernosum [3]. However, for ED caused by DM, PDE5I is not sufficiently effective [4,5]. Alternative remedies, such as vacuum constriction devices, have limitations [6]. Accordingly, a new and effective therapeutic method is urgently needed for DM-associated ED.

Mesenchymal stem cells (MSCs) have long-term self-renewal abilities as well as the capacity to differentiate into a variety of cell types in certain conditions [7]. In different physiological and pathological conditions, MSCs could maintain homeostasis by multi-directional differentiation. They secrete large amounts of cytokines, which carry chemical signals between cells, and are widely used in studies of regenerative medicine [8-10]. Novel MSC-based therapeutic approaches have shown satisfactory treatment effects in clinical applications. MSC therapy is used for the treatment of nerve injury and trauma, inflammatory disease, and transplantation [11-13]. MSCs can promote cellular growth and prevent apoptosis [14,15]. MSC therapy for ED is still in the clinic trial phase [16,17]. Most studies have shown [18-20] that MSC therapy has positive effects on ED. However, the precise effects of MSCs on ED and the mechanisms underlying these effects are unclear [21].

Recent studies [22] have shown that in cavernous-nerve injury-induced ED rats with increased stromal cell-derived factor-1 (SDF-1) expression, ED could be improved. Additionally, Yamaguchi et al. [23] suggested that SDF-1 could drive endothelial progenitor cell recruitment and improve angiogenesis. Accordingly, we believe that an effective method to increase SDF-1 expression in the corpus cavernosum is a potential approach for ED treatment. In the corpus cavernosum of patients with DM-associated ED, there are a number of vascular injuries, including vascular endothelial cell and vascular smooth muscle damage [24-26]. Vascular repair would be improved by sufficient levels of vascular endothelial growth factor (VEGF) in the corpus cavernosum. Additionally, some studies [27] have suggested that VEGF released by MSCs could recover injured vascular tissues. However, other factors explaining the high VEGF expression and the mechanisms by which MSCs in injured tissues promote VEGF expression are unclear.

In this study, we built an ED streptozotocin (STZ)-induced DM rat model. We evaluated the effects and underlying mechanisms of MSC therapy on this DM ED model after SDF-1-expressing bone marrow MSC (SDF-1 eMSC) transplantation. We hypothesized that transplanted SDF-1 eMSCs could increase angiogenesis and thereby improve ED. The main aim of this study was to explore the effects of high SDF-1 expression via BM-MSC therapy and to investigate the relevant mechanisms in the corpus cavernosum of the STZ-induced DM ED rat model.

\section{Results}

\subsection{Measurements of Body Weight and Blood Glucose Levels}

After STZ injection, body weights were significantly lower in DM rats than in Normal rats. After four weeks, the DM ED + SDF-1 eMSC group had significantly greater $(p<0.05)$ weight gain than the DM ED group and significantly lower blood glucose levels $(p<0.05)$ than the DM group (Table 1$)$.

Table 1. Body weights and serum glucose levels.

\begin{tabular}{ccc}
\hline & Pre-DM & After 4 Weeks \\
\hline Body Weight (g) & & \\
Normal $(n=12)$ & $251.6 \pm 8.5$ & $311.3 \pm 13.6$ \\
DM ED $(n=12)$ & $254.1 \pm 9.7$ & $159.6 \pm 16.7^{*}$ \\
DM ED + BM MSC $(n=12)$ & $259.2 \pm 10.9$ & $170.7 \pm 12.8^{*}$ \\
DM ED + SDF-1 eMSC $(n=12)$ & $249.8 \pm 10.3$ & $183.6 \pm 7.2^{*, \#}$ \\
\hline
\end{tabular}


Table 1. Cont.

\begin{tabular}{ccc}
\hline & Pre-DM & After 4 Weeks \\
\hline Serum Glucose $(\mathbf{m g} / \mathbf{d L})$ & & \\
Normal $(n=12)$ & $123.6 \pm 3.3$ & $121.7 \pm 1.9$ \\
DM ED $(n=12)$ & $123.8 \pm 2.9$ & $392.2 \pm 8.7^{*}$ \\
DM ED + BM MSC $(n=12)$ & $124.1 \pm 3.8$ & $383.9 \pm 9.6^{*}$ \\
DM ED + SDF-1 eMSC $(n=12)$ & $122.5 \pm 3.2$ & $376.8 \pm 5.9^{*}, \#$
\end{tabular}

* Significant difference $(p<0.05)$ compared with the Normal group. ${ }^{\#}$ Significant difference $(p<0.05)$ compared with the DM group. The serum glucose levels were measured at fasting status. SDF-1: stromal cell-derived factor-1; DM ED: diabetes mellitus erectile dysfunction.

\subsection{Stromal Cell-derived Factor-1-Expressing Engineered Mesenchymal Stem Cells Significantly Improve} Diabetes Mellitus Erectile Dysfunction

Representative images of intracavernosal pressure (ICP) results are shown in Figure 1. The ICP of the DM ED + BM-MSC group was higher than that of the DM ED group. In a quantitative analysis (Figure 1B), the ICP of the normal group was $0.75 \pm 0.07$, the ICP of the DM ED group was $0.27 \pm 0.08$, the ICP of the DM ED + BM-MSC group was $0.42 \pm 0.11$, and the ICP of the DM ED + SDF- 1 eMSC group was $0.58 \pm 0.11$. These results showed that treatment with BM-MSCs, especially SDF-1 eMSCs, could improve ED. The ICP/MAP ratio was significantly higher in the DM ED + BM-MSC and DM ED + SDF-1 eMSC groups than in the DM ED group $(p<0.05)$.
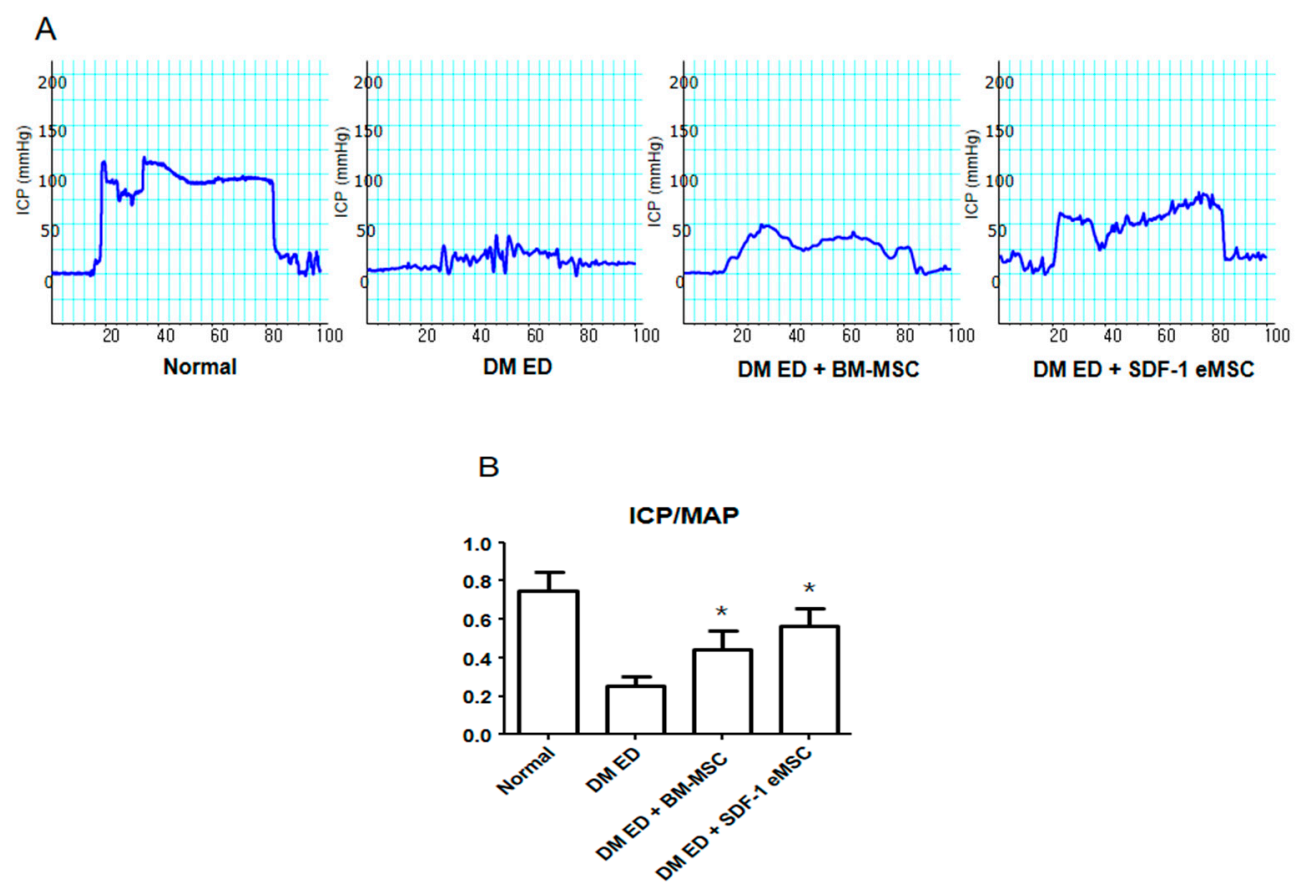

Figure 1. Comparison of erectile function among groups. (A) Representative images of intracavernous pressure (ICP) in response to electrical stimulation of the cavernosal nerve. (B) Ratio of ICP to mean MAP (mean arterial pressure) in each group. Each bar shows the mean value (standard deviation). * $p<0.05$ compared with the DM ED (diabetes mellitus erectile dysfunction) group.

2.3. Stromal Cell-derived Factor-1-Expressing Engineered MSCs Improve the Smooth Muscle Content and Angiogenesis in the Corpus Cavernosum

The smooth muscle and collagen contents in the corpus cavernosum were observed by Masson's trichrome staining. As shown in Figure 2A, the smooth muscle contents were higher in the DM ED + BM-MSC group than in the DM ED group. These results indicated that as the expression of SDF-1 increased, recovery in the ED rats improved. As shown in Figure 3, after the MSC injection, $\alpha$-smooth 
muscle actin ( $\alpha$-SMA) and PECAM expression levels were elevated in the corpus cavernosum, indicating that smooth muscle and angiogenesis increased in injured tissues. Figure 3 shows that in the DM ED + SDF-1 eMSC group, SDF-1-Expressing Engineered MSCs had a more positive influence on tissue repair than BM-MSCs.

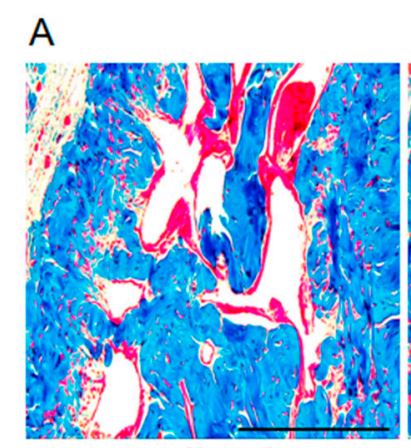

Normal

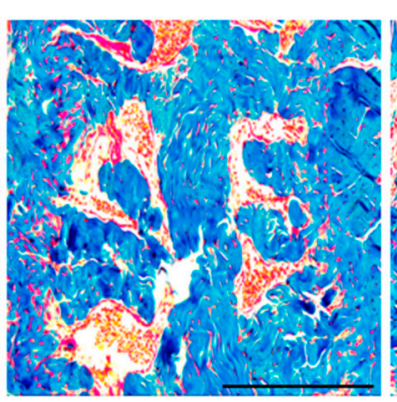

DM ED

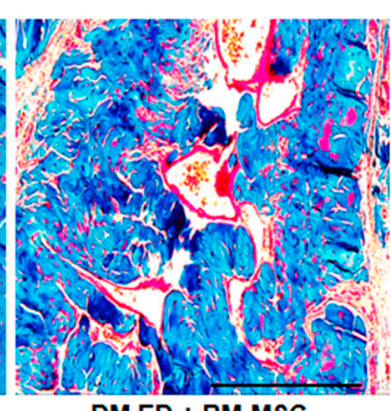

DM ED + BM-MSC

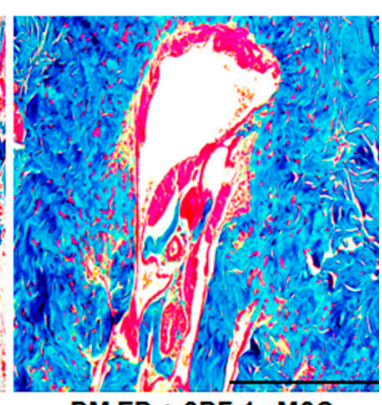

DM ED + SDF-1 eMSC

B

Smooth muscle/Collagen

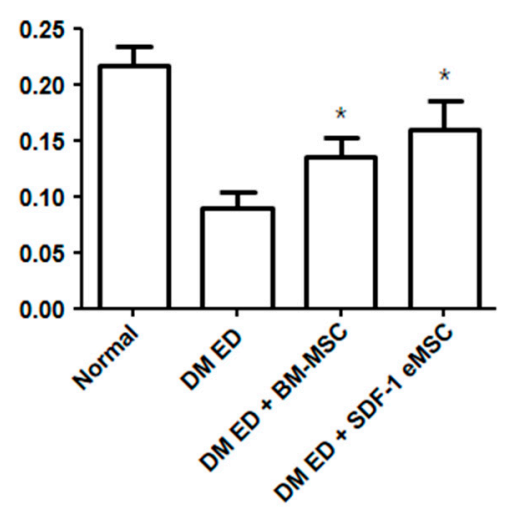

Figure 2. Representative images of Masson Trichrome staining in the corpus cavernosum. (A) Red indicates smooth muscle, and blue represents collagen. Scale bar: $100 \mu \mathrm{m}$. Original magnification: $\times 100$; (B) Percentage area of smooth muscle for each group. Each bar shows the mean value (standard deviation). ${ }^{*} p<0.05$ compared with the DM ED group. 


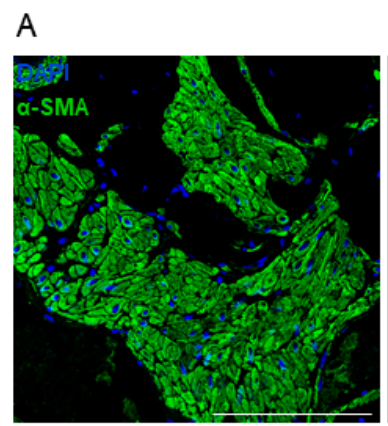

Normal

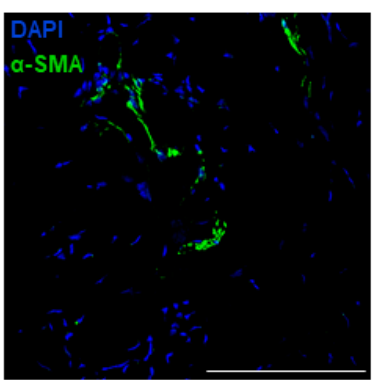

DM ED

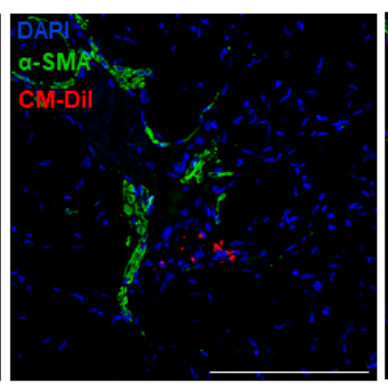

DM ED + BM-MSC

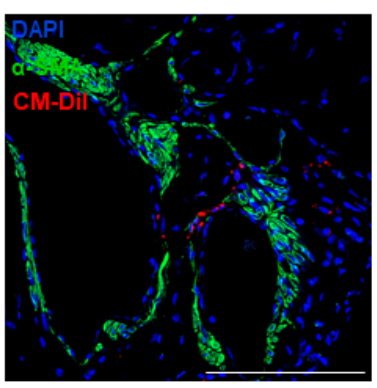

DM ED + SDF-1 eMSC

\section{B}

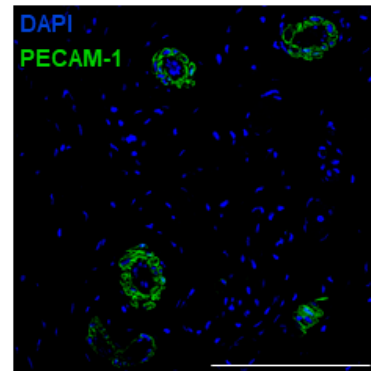

Normal

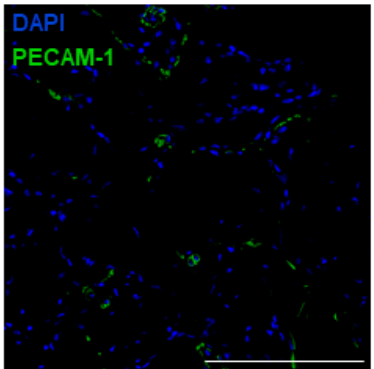

DM ED

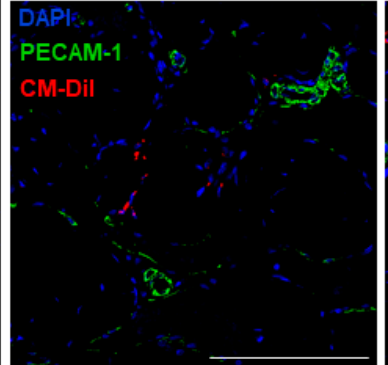

DM ED + BM-MSC

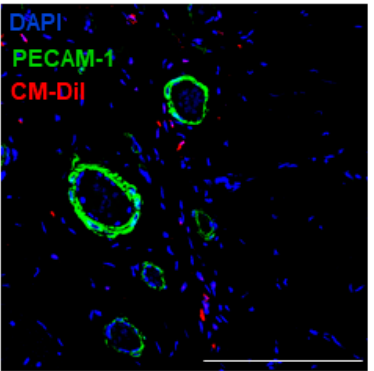

DM ED + SDF-1 eMSC

Figure 3. Representative images of immunofluorescence staining in the corpus cavernosum after treatment with mesenchymal stem cells (MSCs). (A) Representative images of $\alpha$-SMA staining for each group. Red indicates MSCs, green represents $\alpha$-SMA, and blue represents cell nuclei. Scale bar: $100 \mu \mathrm{m}$. Original magnification: $\times 200$; (B) Representative images of PECAM-1 staining for each group. Red indicates MSCs, green is PECAM-1, and blue represents cell nuclei. Original magnification: $\times 200$.

2.4. Increased Expression of neuronal nitric oxide synthase ( $n N O S$ ) and p-eNOS/eNOS in the Dorsal Penile Nerve

Figure $4 \mathrm{~A}$ shows that the intensity of neuronal nitric oxide synthase (nNOS) varied among groups. nNOS was lower in the DM ED group than in the other groups (Figure 4B). In a quantitative analysis, intensities were higher in the SDF-1 eMSC group than in the normal $(p<0.05)$ and BM-MSC groups. Figure 4C summarizes p-eNOS and eNOS expression in each group. Figure 4D shows that in the SDF-1 eMSC group, p-eNOS levels were higher than those in the other groups. 


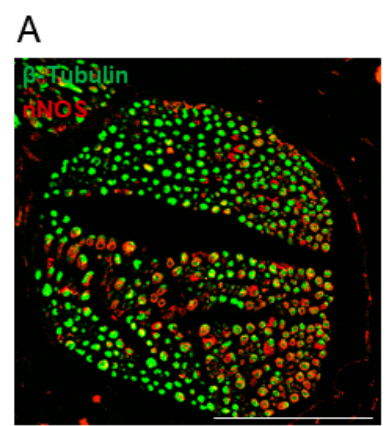

Normal

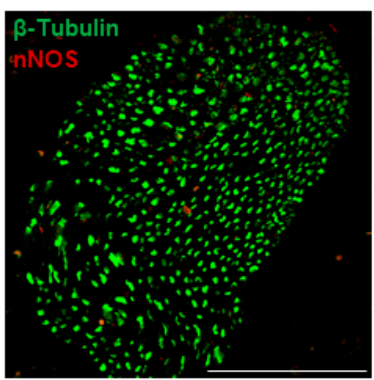

DM ED

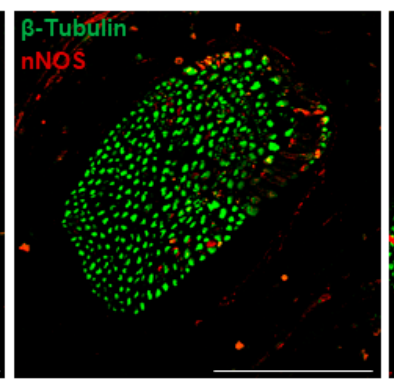

DM ED + BM-MSC

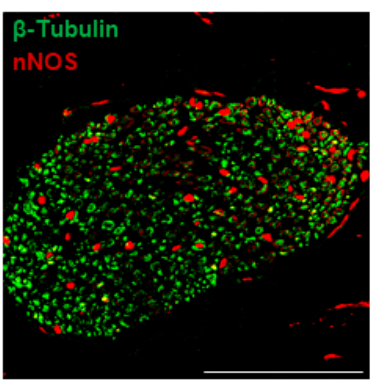

DM ED + SDF-1 eMSC
B

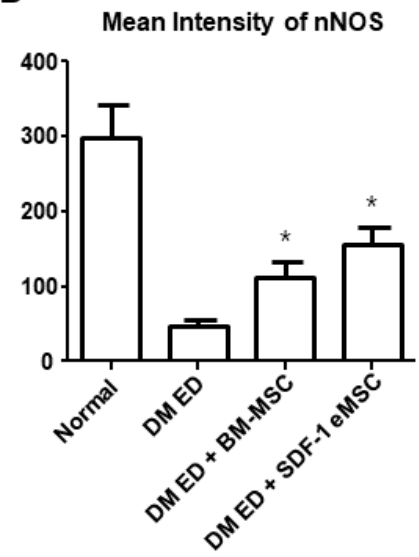

C

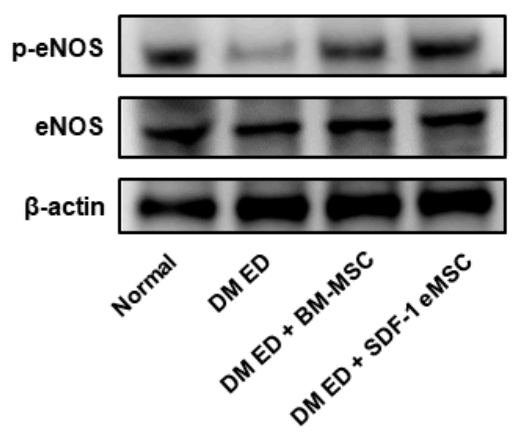

p-eNOS/eNOS

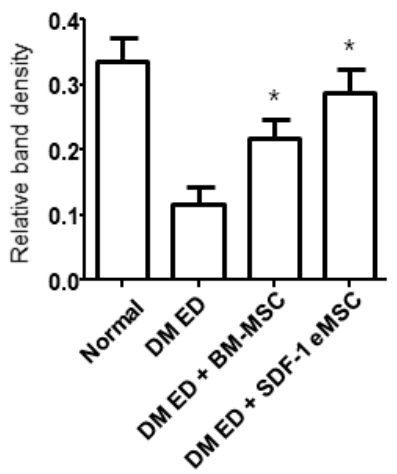

Figure 4. Representative images of neuronal nitric oxide synthase (nNOS) in the dorsal penile nerve. (A) Red indicates nNOS and green indicates the dorsal penile nerve. Scale bar: $100 \mu \mathrm{m}$. Original magnification: $\times 400$; (B) Mean intensity of nNOS for each group; (C) Western blot of phosphorylated endothelial nitric oxide synthase (p-eNOS) and eNOS expression in the corpus cavernosum; (D) Quantitative results of p-eNOS/eNOS in each group. Each bar shows the mean value (standard deviation). $* p<0.05$ compared with the diabetes mellitus erectile dysfunction (DM ED) group.

\subsection{High Stromal Cell-derived Factor-1 Expression Increases Fibroblast Growth Factor and Vascular} Endothelial Growth Factor Levels In Vivo

Figure 5A shows that in the DM ED + SDF-1 eMSC group, SDF-1 expression levels were higher than those of the other groups. Figure 5B summarizes the quantitative analysis $(p<0.05)$. To verify the SDF-1 expression results in each group in vitro, a western blotting analysis of engineered cells and normal cells was performed. As shown in Figure 5C,D, after 10 and 20 passages, the expression of SDF-1 was still higher in the SDF-1 eMSCs group than in the BM-MSC group in vitro $(p<0.05)$. Figure 6 shows that in the two MSC groups, both bFGF and VEGF expression levels were increased. However, in the SDF-1 eMSC group, the expression levels of bFGF and VEGF were higher than those in the BM-MSC group. Though MSC treatment could improve ED, SDF-1 eMSCs were more effective. 
A

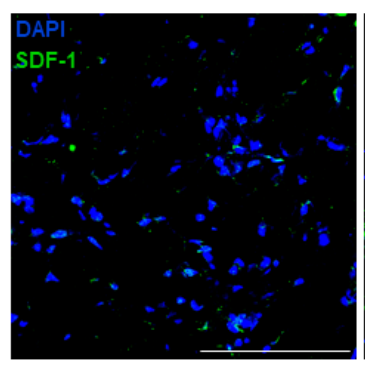

Normal

B

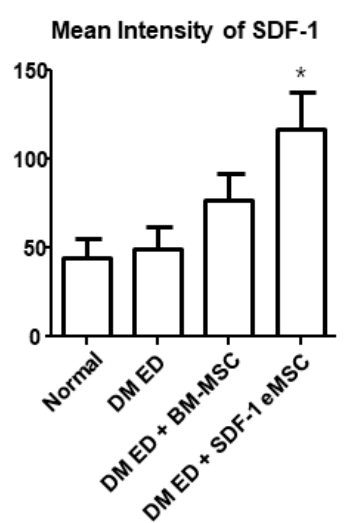

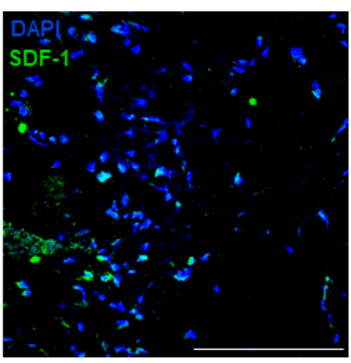

DM ED

\section{C}

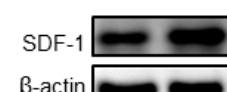

$\beta$-actin
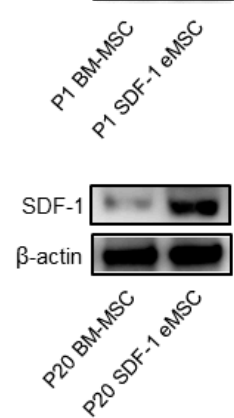

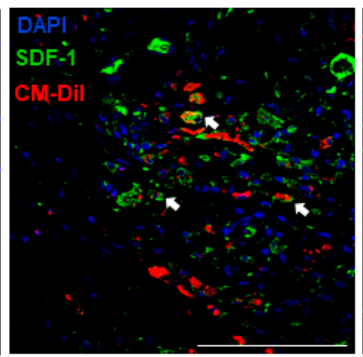

DM ED + BM-MSC

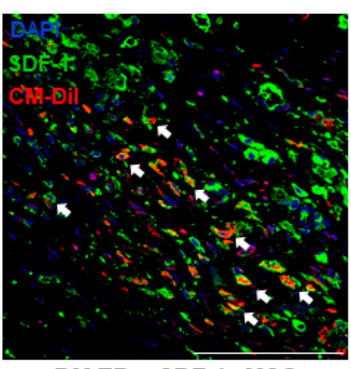

DM ED + SDF-1 eMSC

Figure 5. Representative images of SDF-1 expression in vivo and in vitro. (A) Representative images of SDF-1 expression in the corpus cavernosum. CM-DiI-labeled MSCs are stained in red and green represents SDF-1. Cell nuclei are stained blue with 4,6-diamidino-2-phenylindole. Arrow indicates SDF-1 released by MSCs. Scale bar: $100 \mu \mathrm{m}$. Original magnification: $\times 200$; (B) Mean intensity of SDF-1 expression; (C) SDF-1 expression after each passage of BM-MSCs in vitro; (D) Relative density of bands in the western blot analysis for each group. Each bar shows the mean value (standard deviation). ${ }^{*} p<0.05$ compared with passage $1 \mathrm{BM}-\mathrm{MSCs},{ }^{\#} p<0.05$ compared with passage 10 BM-MSCs, and \& $p<0.05$ compared with passage 20 BM-MSCs. 
A

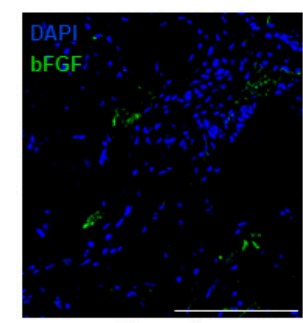

Normal

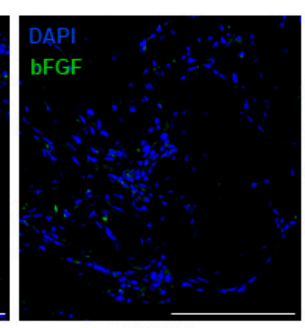

DMED

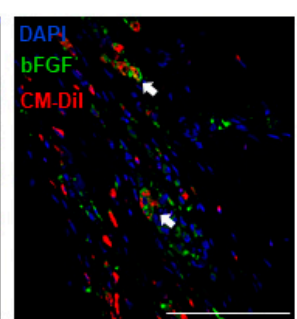

DM ED + BM-MSC

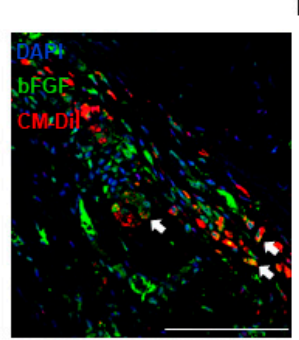

DM ED + SDF-1 eMSC
B

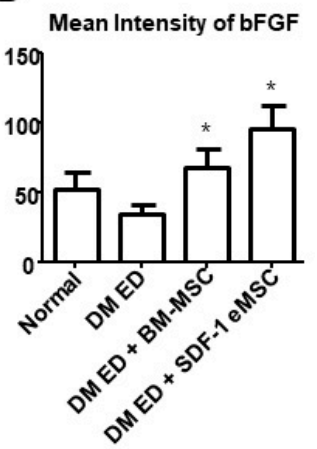

D

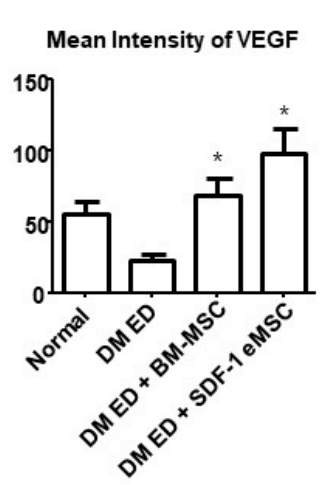

Figure 6. Representative images of bFGF and VEGF expression in the corpus cavernosum. (A) Representative images of bFGF staining for each group. Red indicates MSCs, green indicates bFGF, and blue shows cell nuclei. Arrow indicates bFGF released by MSCs. Scale bar: $100 \mu \mathrm{m}$. Original magnification: $\times 200$; (B) Mean intensity of bFGF expression. (C) Representative images of VEGF staining for each group. Red indicates MSCs, green indicates VEGF, and blue indicates cell nuclei. Arrow indicates VEGF released by MSCs. Scale bar: $100 \mu \mathrm{m}$. Original magnification: $\times 200$; (D) Mean intensity of VEGF expression. Each bar shows the mean value (standard deviation). ${ }^{*} p<0.05$ compared with the DM ED group.

\subsection{MSCs Could Activate the PI3K/AKT Signaling Pathway in the Corpus Cavernosum}

As shown in Figure 7, in the MSC-treated group, p-AKT/AKT levels were higher $(p<0.05)$, indicating greater PI3K/AKT pathway activation compared with that in the DM ED group. Bcl-2 and Bax are downstream effector proteins of the PI3k/AKT pathway. Bcl-2 levels were higher and Bax levels were lower in the MSC-treated group than in the DM ED group $(p<0.05)$. We also observed lower caspase-3 expression in the DM ED + SDF-1 eMSC group, further supporting the reduction in apoptosis in the corpus cavernosum. 
A

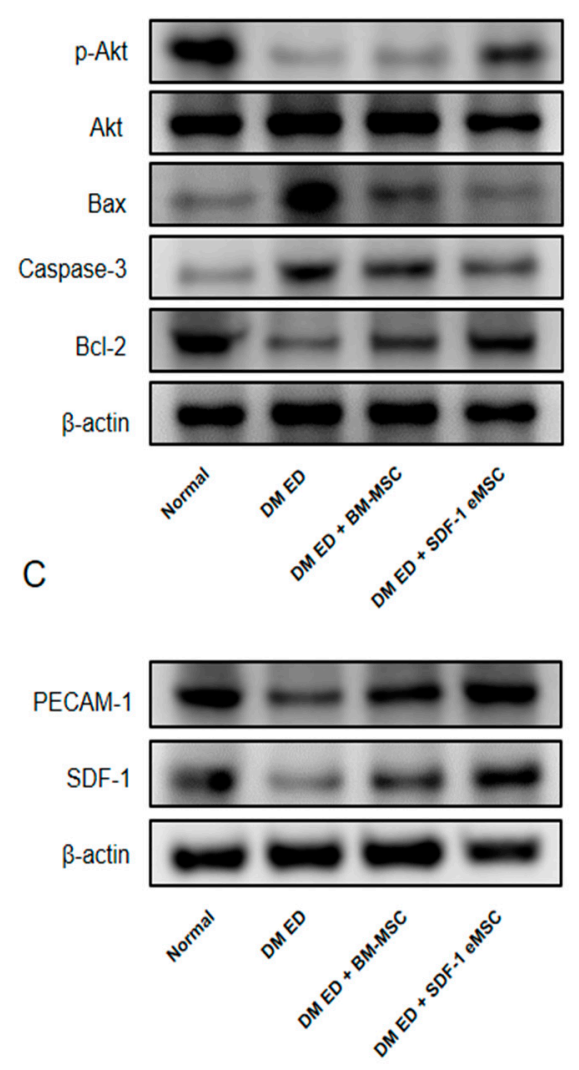

B

D
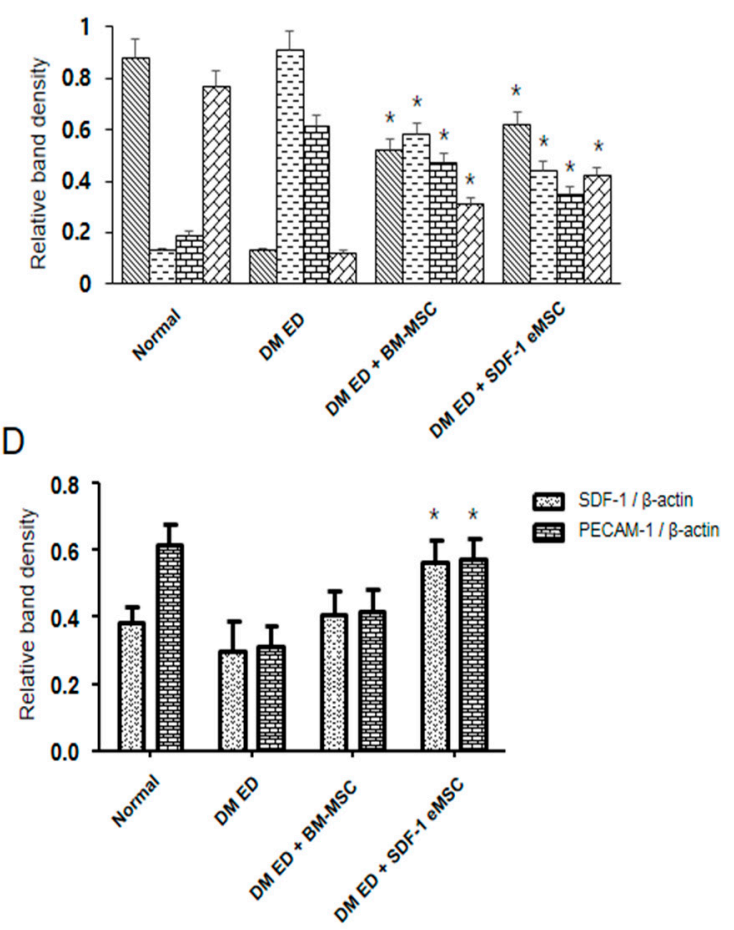

Figure 7. Protein expression in the corpus cavernosum. (A) All groups were compared with respect to $\mathrm{p}-\mathrm{AKT}$, AKT, Bax, Caspase-3, and Bcl-2 expression in the corpus cavernosum by western blotting. (B) Relative density of bands in the western blot analysis for each group. (C) All groups were compared with respect to PECAM-1 and SDF-1 levels in the corpus cavernosum by western blotting. (D) Relative density of bands in the western blot analysis for each group. Each bar shows the mean value (standard deviation). ${ }^{*} p<0.05$ compared with the DM ED group.

\subsection{SDF-1-Expressing Engineered MSC Migration and Angiogenic Activity}

Endothelial cell migration assay (Figure 8A) revealed greater SDF-1 eMSC migration than that of BM-MSCs. For comparison, the assay was repeated using human umbilical vein endothelial cells (HUVECs). As shown in Figure 8A, SDF-1 eMSCs had a similar tendency to HUVECs with respect to cell migration. These results indicated that under high SDF-1 conditions, BM-MSCs generated more mesh than other cells, similar to the results for HUVECs (Figure 8C). 
A
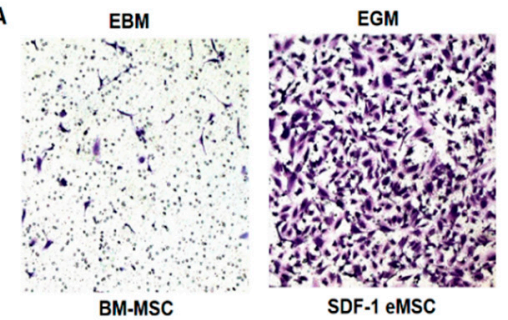
SDF-1 eMSC

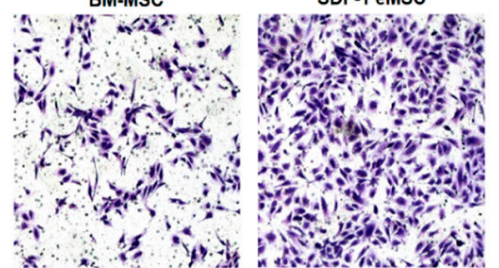

B

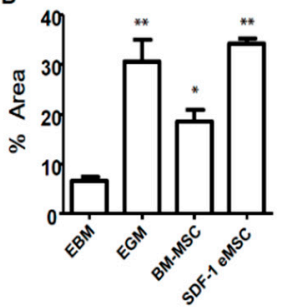

c

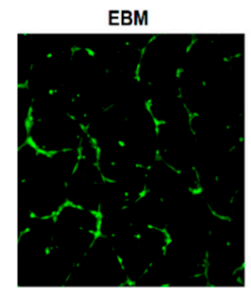

BM-MSC

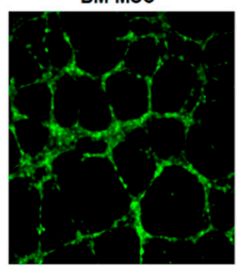

EGM

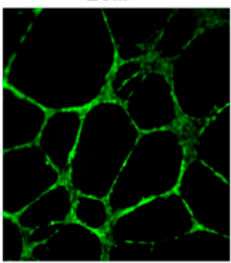

SDF-1 eMSC
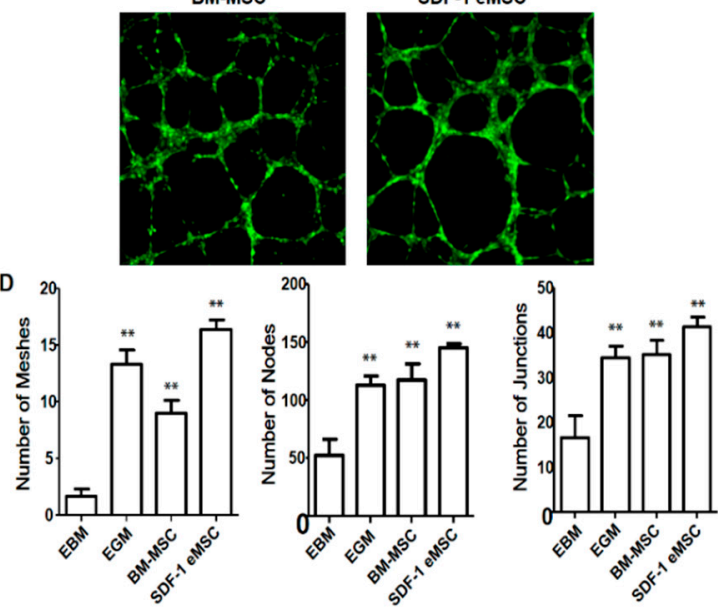

Figure 8. (A) Microscopic fields showing migrated HUVECs at the bottom of the PET membrane; (B) Bar graph representing the percentage of migrated HUVECs per migrated field in response to BM-MSCs and SDF-1 eMSCs. SDF-1 eMSCs had a greater chemotactic effect than BM-MSCs. Original magnification: $\times 100$; (C) Fluorescence microscopy images showing the formation of the tube network on the Matrigel matrix. BM-MSC- and SDF-1 eMSC-treated groups had greater capillary-like structures than EBM-treated groups; (D) Quantitative results for total meshes, total nodes, and total junctions in each group. Original magnification: $\times 50 .{ }^{*} p<0.05$ compared with the DM ED group. ** $p<0.01$ compared with the DM ED group.

\subsection{Expression Levels of SDF-1,VEGF, and bFGF Were Compared between BM-MSCs and Empty Vector} Engineered BM-MSCs

Figure 9 shows that the SDF-1, VEGF, and bFGF were compared between BM-MSCs and empty vector engineered BM-MSCs passage 10. In vitro found no significant $(p<0.05 ; N=6)$ difference as measured by enzyme linked immunosorbent assay (ELISA) and western blotting. 
A

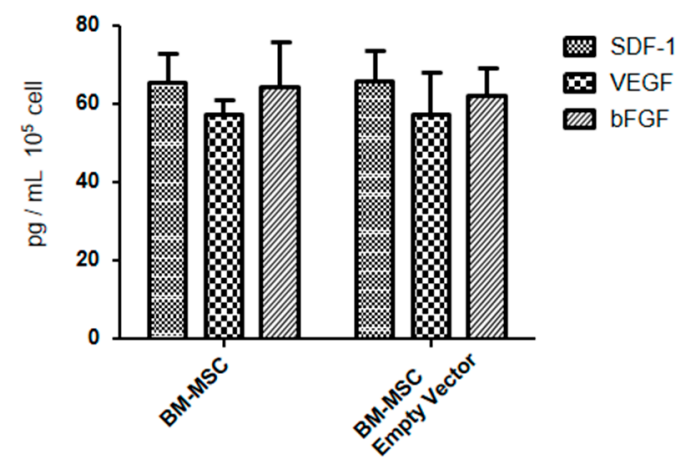

B

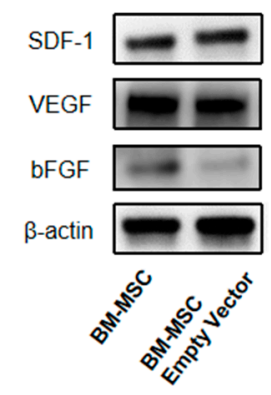

C

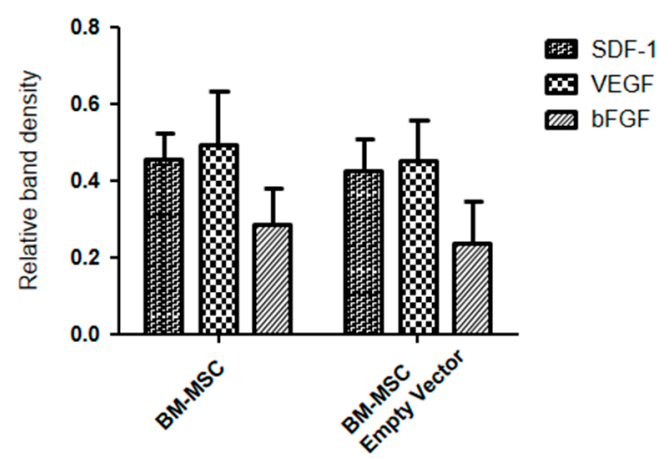

Figure 9. In vitro expression levels of SDF-1, VEGF, and bFGF were compared between BM-MSCs and empty vector engineered BM-MSCs passage 10 by quantitative ELISA and western blotting. (A) SDF-1, VEGF, and bFGF concentration in vitro by ELISA; (B) Expression levels of SDF-1, VEGF, and bFGF in vitro by western blotting; (C) Relative density of bands in the western blot analysis for each group.

\section{Discussion}

DM ED is generally treated by drug therapy, but a considerable portion of patients are not satisfied with this approach [28]. Accordingly, an alternative approach is needed. ED caused by DM is related to blood vessel spasms and vascular injury due to chronic hyperglycemia $[25,26,28,29]$ in the corpus cavernosum. Therefore, treatment of vascular injury is the key for improving DM ED. Certainly, ED is not a life-threatening disease; therefore, it is difficult to apply genetically modified stem cells in ED patients. Nevertheless, urologists are still striving to find an effective method to address this issue. Recently, Yiou et al. [20] reported a clinical trial that investigated extracavernous injection of MSCs into patients with ED. Stem cells were aspirated from the posterior iliac crest under general anesthesia and injected into both cavernous bodies on the same day. After a 1-year follow-up, they concluded that MSC injection may improve erectile function, which boosted our confidence. A recent study has shown that MSC administration increases VEGF in targeted tissues [27]; the increase in VEGF was attributed to the release of MSCs. However, we wondered whether other factors led to high VEGF expression and why MSCs in injured tissues produced more VEGF. In this study, we found that compared with the BM-MSC group, rats with SDF-1-expressing BM-MSCs had increased vascular smooth muscle contents in the corpus cavernosum. Similar results were obtained in an ICP analysis, indicating that SDF-1 was an important factor in injured tissue recovery and ED improvement.

Liang et al. [30] suggested that the main limitation of MSC treatment was the inability of transplanted MSCs to accurately accumulate at the therapeutic target and function effectively. A previous study [22] has shown that injected MSCs migrate to the circulatory system and disperse via the blood flow; only a few MSCs gather at injured tissues, below the targeted therapeutic concentrations. By labelling MSCs with a fluorescent dye, Cell Tracker ${ }^{\mathrm{TM}} \mathrm{CM}$-DiI [31], we found that after injection, a large number of MSCs gathered in the corpus cavernosum in the treatment group, proving that MSCs migrated to the target tissues. Studies have shown that SDF-1 levels are high during cell 
proliferation and tissue repair [30,32], and these chemotactic factors could promote the accumulation of these functional cells at injured tissues [33]. In our study, we showed that BM-MSCs expressing high levels of SDF-1 could release more SDF-1 than normal BM-MSCs. We found increased VEGF expression and angiogenesis in the upgraded SDF-1-expressing BM-MSC group compared with the other groups, explaining why ED rats recovered more effectively after SDF-1-expressing BM-MSC treatment. Fandel et al. [22] showed that in cavernous nerve-injured ED rats, SDF-1 could drive injected stem cells to the major pelvic ganglia for neural restoration. Yamaguchi et al. [23] found that SDF-1 could drive endothelial progenitor cells to injured tissues and improve angiogenesis.

Chemotaxis of SDF-1 could drive both exogenous and endogenous stem cell recruitment. Thus, VEGF expression in injured tissues should be greater than that in MSCs, and other endogenous stem cells could gather at the targeted tissues for vascular repair. We found differential expression of SDF-1 among the groups. In the SDF-1-expressing BM-MSC group, VEGF expression was higher than that in the normal MSC group. Since the MSCs were equal and VEGF differed between the two groups, we inferred that SDF-1 played a key role in the process of ED improvement. These results indicated that SDF-1 expressed by the injected BM-MSCs drove endogenous cells to targeted tissues. These endogenous cells could accelerate angiogenesis and tissue repair by releasing VEGF, as confirmed by our results.

We performed preliminary studies to verify whether or not empty vector engineered MSCs differed from un-engineered MSCs regarding their ability to affect and influence the outcome of our in vivo results. We tested these cells for the production of SDF-1, VEGF, and bFGF in vitro and found no significant difference as measured by ELISA and western blotting. In addition, we performed western blot analysis for the same factors and found no difference with the exception that bFGF was slightly lower in the empty vector engineered BM-MSCs. From these findings, we determined that un-engineered MSCs could serve as a good control, and thus, it was not necessary to continue studying empty vector engineered MSCs as a control in our in vivo studies. Furthermore, previous studies showed that empty vector engineered adipose stem cells had no notable effect on chondrogenesis [34].

De Bock et al. [35] suggested that glycolysis provided energy to endothelial cells for proliferation, even in conditions of sufficient oxygen. In new tissues, vascular growth was promoted by continuous budding. At the end of the budding, the blood supply was insufficient to support angiogenesis, and endothelial cells obtained ATP via anaerobic glycolysis. Similarly, in the corpus cavernosum of DM ED, vascular injury and vasospasm caused by hyperglycemia lead to an insufficient blood supply. However, endothelial cells still obtain enough ATP to support proliferation for vascular repair via anaerobic glycolysis. Han et al. [36] believed that p-AKT could stimulate endothelial cells to produce substantial eNOS, which was the key factor for vasodilatation. In this study, we found higher levels of eNOS in the corpus cavernosum of the treatment group than in the untreated group, and p-AKT/AKT showed a similar trend. It is possible that MSCs could produce high levels of VEGF in the corpus cavernosum, which combined with the surface receptors of endothelial cells to trigger the PI3K-AKT signaling pathway, thereby increasing eNOS. A large quantity of eNOS would stimulate vasodilatation and inhibit vasospasm, which could recover the blood supply and improve ED.

Stem cell therapy is beneficial for ED caused by nerve injury [37]. Stem cells could promote nerve recovery. nNOS produced by nerve cells is not only involved in the penis erection reflex but also plays key roles in angiogenesis and vasodilatation [38]. Wang et al. [39] found that MSCs could stimulate nerve cells to produce nNOS, which improved angiogenesis in the injured tissues. In this study, we obtained similar results; in the corpus cavernosum of the treatment group, nNOS levels were higher than those in the untreated group, indicating that MSCs could promote nerve cell production of nNOS in vivo for angiogenesis. After MSC transplantation, hypoxemia and the oxidative stress response caused by hyperglycemia decreased the survival rate of injected MSCs, and the short lifespan of MSCs after intravenous infusion raised doubt about the contribution of growth factors secreted by MSCs to the modulation of vascular repair and cellular proliferation [40,41]. Bcl-2 could inhibit apoptosis, and downgraded Bax could decrease the rate of apoptosis [42]. In our study, we found 
activation of the PI3K-AKT pathway in the corpus cavernosum, which was thought to stimulate the expression of Bcl-2 and inhibit the expression of Bax. We found significant Bcl-2 and Bax expression in the corpus cavernosum in all groups. We inferred that the activation of the PI3K-AKT pathway by VEGF increased the expression of Bcl-2 and decreased that of Bax, which improved the survival rate of the transplanted MSCs.

We further tested upstream factors and downstream effector proteins, which did not block the signaling pathway, to verify our findings.

\section{Materials and Methods}

\subsection{SDF-1-Expressing MSCs and Experimental Animal Preparation}

Primary bone marrow mesenchymal stem cells (BM-MSCs) were cultured in low glucose-containing Dulbecco's modified Eagle's medium (Gibco, Gaithersburg, MD, USA) supplemented with $20 \%$ fetal bovine serum (FBS; Gibco) and $5 \mathrm{ng} / \mathrm{mL}$ basic fibroblast growth factor (bFGF; Cell Signaling Technology, Danvers, MA, USA) at $37^{\circ} \mathrm{C}$ and $5 \% \mathrm{CO}_{2}$, and engineered BM-MSCs were cultured with $10 \%$ FBS.

To generate engineered BM-MSCs, c-myc, hTERT, tetracycline transactivator ( $t T A)$, and SDF-1 genes were synthesized and transfected using the pBD lentiviral vector (SL BIGEN, Seongnam, Korea). Transfected engineered BM-MSCs were selected by antibiotics. Selected engineered BM-MSCs were isolated to obtain a monoclonal cell population by the limiting dilution method. Final monoclonal cells were selected based on SDF-1 expression, proliferation, and other MSC phenotypes. Before in vivo injection, SDF-1-expressing engineered BM-MSCs (SDF-1 eMSCs) were irradiated.

The reference sequence for SDF-1 was NM_000609.6. We introduced an optimized DNA sequence into the vector (GenScript, Nanjing, China). DNA sequencing was performed to confirm that the reading frame of the introduced sequence was correct (Cosmo Genetech, Seoul, Korea). Cryopreserved cells were thawed and seeded onto 12-well plates at a density of $1 \times 10^{5}$ cells per well. After $48 \mathrm{~h}$, the supernatant was harvested and SDF-1 protein levels were measured by ELISA (R\&D Systems, Minneapolis, MN, USA); the expression level of SDF-1 was about $10 \mathrm{ng} / \mathrm{mL}$.

Fifty 8-week-old male Sprague-Dawley rats weighing about 250-300 g were purchased from Orient Bio Co. (Seongnam, Korea). All animal experiments in this study were approved by the Institutional Animal Care and Use Committee of the Catholic University of Korea (CUMC-2016-0218-01, 31 August 2016). All surgeries were performed under chloral hydrate anesthesia, and all efforts were made to minimize animal suffering.

\subsection{Establishment of DM ED Models and BM-MSC Administration}

After fasting, 12 rats were randomly chosen for the normal group (control) and intraperitoneally injected with saline. In the other rats, ED was induced by an intraperitoneal injection of streptozotocin (STZ; Sigma Aldrich, St. Louis, MO, USA) at a dose of $60 \mathrm{mg} / \mathrm{kg}$ body weight. At $72 \mathrm{~h}$ after STZ injection, DM was confirmed by blood glucose levels; blood samples were obtained from tail veins, and only rats with a fasting blood glucose level of $\geq 300 \mathrm{mg} / \mathrm{dL}$ were selected as diabetic and used for further tests $(n=36)$. The rats were randomly divided into 4 groups: a normal group (control, $n=12$ ), a DM ED group $(n=12)$, a DM ED + BM-MSC group $(n=12)$, and a DM ED + SDF-1 eMSC group $(n=12)$.

\subsection{BM-MSC Treatment}

Four weeks after DM ED was induced, rats in the DM ED + BM-MSC group or DM ED + SDF-1 eMSC group were treated with BM-MSCs or SDF-1 eMSCs by bilateral intracavernous injection under anesthesia $\left(1 \times 10^{6} \mathrm{BM}\right.$-MSCs diluted in phosphate-buffered saline). Rats in the control group were injected with an equal volume of saline. To track the location of BM-MSCs, they were labeled with 
a fluorescent dye (Cell Tracker ${ }^{\mathrm{TM}}$ CM-DiI; Molecular Probes, Eugene, OR, USA) according to the manufacturer's protocol.

\subsection{Measurement of Erectile Function}

After treatment for 4 weeks, all rats were tested for erectile function by measuring intracavernous pressure (ICP) and mean arterial pressure (MAP) under anesthesia. A 23-gauge butterfly needle containing $250 \mathrm{U} / \mathrm{mL}$ heparin solution was carefully inserted into the proximal corpus cavernosum and the other end of the PE-50 tube was connected to a pressure transducer (Grass model S48 K; Astro-Med Inc., West Warwick, RI, USA) to measure the ICP. A bipolar stainless steel electrical stimulator was used to stimulate the major pelvic ganglion at $10 \mathrm{~V}$ for $50 \mathrm{~s}$ and $2.4 \mathrm{~mA}$ with a pulse width of $2.5 \mathrm{~ms}$. The maximum ICP value of three stimulations was used for the statistical analysis in each rat. ICP was normalized to MAP, which was recorded using a BD Intramedic PE-50 tubing (BD, Franklin Lakes, NJ, USA) inserted into the left carotid artery at the same time. After the measurement of erectile function, rats were sacrificed and the penises were harvested for immunohistochemistry and western blot analyses.

\subsection{Histochemistry}

The collected cavernous nerve and penis samples were fixed in $4 \%$ paraformaldehyde for $24 \mathrm{~h}$ at $4{ }^{\circ} \mathrm{C}$ before creating a paraffin block. The following primary antibodies were used: Neuronal nitric oxide synthase (nNOS, diluted 1:200; Santa Cruz Biotechnologies, Santa Cruz, CA, USA), alpha smooth muscle actin ( $\alpha$-SMA, diluted 1:500; Abcam, Cambridge, UK), vascular endothelial growth factor (VEGF; diluted 1:200; Santa Cruz Biotechnologies), basic fibroblast growth factor (bFGF, diluted 1:500; Cell Signaling Technology), stromal cell-derived factor-1 (SDF-1 diluted 1:200; Abcam), and platelet endothelial cell adhesion molecule (PECAM-1, diluted 1:500; Abcam), and 6-diamidino-2-phenylindole (DAPI; Vector Laboratories, Inc., Burlingame, CA, USA) was used to stain nuclei. Digital images were obtained using a Zeiss LSM 510 Meta confocal microscope (Zeiss, Oberkochen, Germany), and the mean intensity was calculated using ZEN 2012 (Zeiss).

\subsection{Western Blotting}

The collected corpus cavernosum tissue was homogenized using ice-cold RIPA buffer (Cell Signaling Technology) containing ethylene diamine tetra acetic acid-free protease inhibitor cocktail and phosphatase inhibitor cocktail (Roche Diagnostics $\mathrm{GmbH}$, Basel, Switzerland), and particulate mass was removed by centrifugation $(15,000 \times g)$ for $15 \mathrm{~min}$ at $4{ }^{\circ} \mathrm{C}$. Supernatants were analyzed by SDS-PAGE. Primary antibodies against endothelial nitric oxide synthase (eNOS diluted 1:200; Cell Signaling Technology), phosphorylated endothelial nitric oxide synthase (p-eNOS diluted 1:200; Cell Signaling Technology), AKT (diluted 1:200; Cell Signaling Technology), p-AKT (diluted 1:200; Cell Signaling Technology), Bcl-2 (diluted 1:200; Cell Signaling Technology), Bax (diluted 1:200; Cell Signaling Technology), SDF-1 (diluted 1:500; Abcam), PECAM (diluted 1:200; Abcam), Caspase-3 (diluted 1:400; Cell Signaling Technology), VEGF (diluted 1:500; Abcam), bFGF (diluted 1:500; Abcam), and $\beta$-actin (diluted 1:1000; Abcam) were used.

\subsection{BM-MSC and SDF-1 eMSC Preparation in Conditioned Media}

BM-MSCs and SDF-1 eMSCs were seeded in a $100 \mathrm{~mm}$ culture dish. After fully attaching, completed growth media were removed and cells were washed with PBS 3 times. Subsequently, $10 \mathrm{~mL}$ of fresh serum-free basal medium was added, followed by incubation at $37^{\circ} \mathrm{C}$ in $5 \% \mathrm{CO}_{2}$ for 3 days. Collected media were centrifuged at $3000 \mathrm{rpm}$ at $4{ }^{\circ} \mathrm{C}$ for $15 \mathrm{~min}$. The supernatant was collected and SDF-1 eMSC conditioned media were stored at $-80^{\circ} \mathrm{C}$ until further analyses. 


\subsection{Tube Formation Assay}

A total of $200 \mu \mathrm{L}$ of BD Growth Factor Reduced (GFR) Matrigel Basement Membrane Matrix (BD Biosciences) was added to a 24-well plate and incubated at $37{ }^{\circ} \mathrm{C}$ for $30 \mathrm{~min}$. HUVECs $\left(1 \times 10^{5}\right.$ cells/well) were incubated on a plate coated with Matrigel in endothelial growth media (EGM), endothelial basal media (EBM) (no growth supplement), and EBM with 30\% conditioned media (BM-MSC and SDF-1 eMSC). After $12 \mathrm{~h}$, cells were stained using calcein AM dye $(2 \mu \mathrm{g} / \mathrm{mL})$. Tube formation was observed using a fluorescence microscope (Axio200; Carl Zeiss). Images were traced and skeletonized using an image and angiogenesis tool. The total number of meshes, nodes, and junctions were quantified for each skeleton.

\subsection{Endothelial Cell Migration Assay}

The spontaneous formation of capillary-like structures by HUVECs on the Matrigel basement membrane matrix was evaluated to assess angiogenesis. A total of $5 \times 10^{4} \mathrm{HUVECs}$ in $400 \mu \mathrm{L}$ of EBM (no growth supplement) were plated in the upper compartment, and $600 \mu \mathrm{L}$ of medium was added to the lower chamber (EGM, EBM, EBM with BM-MSC, and SDF-1 eMSC conditioned media). The plates were then cultured for $8 \mathrm{~h}$ under normal conditions. To estimate the number of migrated cells, the inserts were fixed with $4 \%$ PFA and the upper surface of the Transwell membranes was gently swabbed with cotton to remove non-migrated cells and stained with crystal violet $(0.1 \%)$. The cells that traversed the membrane were counted by bright-field microscopy.

\subsection{ELISA}

The concentration of SDF-1, VEGF and bFGF were measured by species-specific immunoassay ELISA kits (R\&D Systems Europe, Abingdon, UK) according to the manufacturer's instructions. BM-MSCs and empty vector engineered BM-MSCs at passage 10 were cultured for $12 \mathrm{~h}$ in a constant-temperature incubator, and then $1 \mathrm{~mL}$ of the medium was collected. Absorbance was read at a wavelength of $450 \mathrm{~nm}$ using a microplate reader (Synergy H1 M, Biotek, Winooski, VT, USA).

\subsection{Statistical Analysis}

All data are presented as means \pm standard error (SD) and were analyzed using SPSS version 22.0 (IBM, Armonk, NY, USA). Student's $t$-tests, one-way ANOVA, and $2 \times 2$ factorial ANOVA were used, as appropriate, to evaluate differences among groups. $p<0.05$ was considered statistically significant.

\section{Conclusion}

Our results proved that SDF-1 eMSCs released chemotactic factors, which could drive MSCs to migrate to targeted tissues, thereby increasing angiogenesis and effectively improving ED. Additionally, high SDF-1 expression substantially decreased apoptosis in the corpus cavernosum.

Data Availability: The data used to support the conclusions of this study are available from the corresponding author upon request.

Author Contributions: Conceptualization, S.H.J.; Methodology, S.H.J. and G.Q.Z.; Software, S.H.J.; Validation, W.J.B., S.W.C., H.C.J., H.J.C., U.-S.H., S.-H.H., J.Y.L., E.B.K. and S.W.K.; Formal Analysis, S.H.J. and G.Q.Z.; Investigation, S.H.J. and W.J.B.; Resources, H.-J.K., S.M.L. and H.-Y.K.; Writing-Original Draft Preparation, S.H.J.; Writing-Review \& Editing, S.H.J., G.Q.Z. and S.W.K.; Visualization, S.H.J.; Supervision, S.W.K.; Project Administration, W.J.B. and S.W.K.; Funding Acquisition, S.W.K.

Funding: This research was funded by the Ministry of Science and ICT (NRF-2018M3A9E8020861).

Acknowledgments: This research was supported by the Bio and Medical Technology Development Program of the National Research Foundation (NRF) funded by the Ministry of Science and ICT (NRF-2018M3A9E8020861). This study was supported by Research Fund of Seoul St. Mary's Hospital, The Catholic University of Korea.

Conflicts of Interest: The authors declare no conflicts of interest. 


\section{References}

1. Cheng, J.Y.; Ng, E.M.; Ko, J.S.; Chen, R.Y. Physical activity and erectile dysfunction: Meta-analysis of population-based studies. Int. J. Impot. Res. 2007, 19, 245-252. [CrossRef] [PubMed]

2. Bacon, C.G.; Hu, F.B.; Giovannucci, E.; Glasser, D.B.; Mittleman, M.A.; Rimm, E.B. Association of type and duration of diabetes with erectile dysfunction in a large cohort of men. Diabetes Care 2002, 25, 1458-1463. [CrossRef] [PubMed]

3. Peak, T.C.; Anaissie, J.; Hellstrom, W.J. Current perspectives on stem cell therapy for erectile dysfunction. Sex. Med. Rev. 2016, 4, 247-256. [CrossRef] [PubMed]

4. Rendell, M.S.; Rajfer, J.; Wicker, P.A.; Smith, M.D. Sildenafil for treatment of erectile dysfunction in men with diabetes: A randomized controlled trial. Sildenafil Diabetes Study Group. JAMA 1999, 281, 421-426. [CrossRef] [PubMed]

5. Bai, G.Y.; Zhou, F.; Hui, Y.; Xu, Y.D.; Lei, H.E.; Pu, J.X.; Xin, Z.C. Effects of Icariside II on corpus cavernosum and major pelvic ganglion neuropathy in streptozotocin-induced diabetic rats. Int. J. Mol. Sci. 2014, 15, 23294-23306. [CrossRef] [PubMed]

6. Raina, R.; Agarwal, A.; Ausmundson, S.; Lakin, M.; Nandipati, K.C.; Montague, D.K.; Mansour, D.; Zippe, C.D. Early use of vacuum constriction device following radical prostatectomy facilitates early sexual activity and potentially earlier return of erectile function. Int. J. Impot. Res. 2006, 18, 77-81. [CrossRef] [PubMed]

7. Bunpetch, V.; Wu, H.; Zhang, S.; Ouyang, H. From "Bench to Bedside": Current Advancement on Large-Scale Production of Mesenchymal Stem Cells. Stem Cells Dev. 2017, 26, 1662-1673. [CrossRef] [PubMed]

8. Carlsson, P.O.; Schwarcz, E.; Korsgren, O.; le Blanc, K. Preserved $\beta$-cell function in type 1 diabetes by mesenchymal stromal cells. Diabetes 2015, 64, 587-592. [CrossRef] [PubMed]

9. Uccelli, A.; Moretta, L.; Pistoia, V. Mesenchymal stem cells in health and disease. Nat. Rev. Immunol. 2008, 8 , 726-736. [CrossRef] [PubMed]

10. Song, L.; Sun, Z.; Kim, D.S.; Gou, W.; Strange, C.; Dong, H.; Cui, W.; Gilkeson, G.; Morgan, K.A.; Adams, D.B.; et al. Adipose stem cells from chronic pancreatitis patients improve mouse and human islet survival and function. Stem Cell Res. Ther. 2017, 8, 192. [CrossRef] [PubMed]

11. Meirelles Lda, S.; Nardi, N.B. Murine marrow-derived mesenchymal stem cell: Isolation, in vitro expansion, and characterization. Br. J. Haematol. 2003, 123, 702-711. [CrossRef] [PubMed]

12. Le Blanc, K.; Frassoni, F.; Ball, L.; Locatelli, F.; Roelofs, H.; Lewis, I.; Lanino, E.; Sundberg, B.; Bernardo, M.E.; Remberger, M.; et al. Mesenchymal stem cells for treatment of steroid-resistant, severe, acute graft-versus-host disease: A phase II study. Lancet 2008, 371, 1579-1586. [CrossRef]

13. Reinders, M.E.; de Fijter, J.W.; Roelofs, H.; Bajema, I.M.; de Vries, D.K.; Schaapherder, A.F.; Claas, F.H.; van Miert, P.P.; Roelen, D.L.; van Kooten, C.; et al. Autologous bone marrow-derived mesenchymal stromal cells for the treatment of allograft rejection after renal transplantation: Results of a phase I study. Stem Cells Transl. Med. 2013, 2, 107-111. [CrossRef] [PubMed]

14. Schive, S.W.; Mirlashari, M.R.; Hasvold, G.; Wang, M.; Josefsen, D.; Gullestad, H.P.; Korsgren, O.; Foss, A.; Kvalheim, G.; Scholz, H. Human Adipose-Derived Mesenchymal Stem Cells Respond to Short-Term Hypoxia by Secreting Factors Beneficial for Human Islets In Vitro and Potentiate Antidiabetic Effect In Vivo. Cell Med. 2017, 9, 103-116. [CrossRef] [PubMed]

15. Peng, K.Y.; Liu, Y.H.; Li, Y.W.; Yen, B.L.; Yen, M.L. Extracellular matrix protein laminin enhances mesenchymal stem cell (MSC) paracrine function through $\alpha \mathrm{v} \beta 3 / \mathrm{CD} 61$ integrin to reduce cardiomyocyte apoptosis. J. Cell. Mol. Med. 2017, 21, 1572-1583. [CrossRef] [PubMed]

16. Mangir, N.; Turkeri, L. Stem cell therapies in post-prostatectomy erectile dysfunction: A critical review. Can. J. Urol. 2017, 24, 8609-8619. [PubMed]

17. Soebadi, M.A.; Milenkovic, U.; Weyne, E.; Castiglione, F.; Albersen, M. Stem cells in male sexual dysfunction: Are we getting somewhere? Sex. Med. Rev. 2017, 5, 222-235. [CrossRef] [PubMed]

18. Bochinski, D.; Lin, G.T.; Nunes, L.; Carrion, R.; Rahman, N.; Lin, C.S.; Lue, T.F. The effect of neural embryonic stem cell therapy in a rat model of cavernosal nerve injury. BJU Int. 2004, 94, 904-909. [CrossRef] [PubMed] 
19. Bivalacqua, T.J.; Deng, W.; Kendirci, M.; Usta, M.F.; Robinson, C.; Taylor, B.K.; Murthy, S.N.; Champion, H.C.; Hellstrom, W.J.; Kadowitz, P.J. Mesenchymal stem cells alone or ex vivo gene modified with endothelial nitric oxide synthase reverse age-associated erectile dysfunction. Am. J. Physiol. 2007, 292, H1278-H1290. [CrossRef] [PubMed]

20. Yiou, R.; Hamidou, L.; Birebent, B.; Bitari, D.; Lecorvoisier, P.; Contremoulins, I.; Khodari, M.; Rodriguez, A.M.; Augustin, D.; Roudot-Thoraval, F.; et al. Safety of Intracavernous Bone Marrow-Mononuclear Cells for Postradical Prostatectomy Erectile Dysfunction: An Open Dose-Escalation Pilot Study. Eur. Urol. 2016, 69, 988-991. [CrossRef] [PubMed]

21. Yiou, R.; Mahrouf-Yorgov, M.; Trebeau, C.; Zanaty, M.; Lecointe, C.; Souktani, R.; Zadigue, P.; Figeac, F.; Rodriguez, A.M. Delivery of human mesenchymal adipose-derived stem cells restores multiple urological dysfunctions in a rat model mimicking radical prostatectomy damages through tissue-specific paracrine mechanisms. Stem Cells 2016, 34, 392-404. [CrossRef] [PubMed]

22. Fandel, T.M.; Albersen, M.; Lin, G.; Qiu, X.; Ning, H.; Banie, L.; Lue, T.F.; Lin, C.S. Recruitment of intracavernously injected adipose-derived stem cells to the major pelvic ganglion improves erectile function in a rat model of cavernous nerve injury. Eur. Urol. 2012, 61, 201-210. [CrossRef] [PubMed]

23. Yamaguchi, J.; Kusano, K.F.; Masuo, O.; Kawamoto, A.; Silver, M.; Murasawa, S.; Bosch-Marce, M.; Masuda, H.; Losordo, D.W.; Isner, J.M.; et al. Stromal cell-derived factor-1 effects on ex vivo expanded endothelial progenitor cell recruitment for ischemic neovascularization. Circulation 2003, 107, 1322-1328. [CrossRef] [PubMed]

24. Vlachopoulos, C.; Rokkas, K.; Ioakeimidis, N.; Stefanadis, C. Inflammation, metabolic syndrome, erectile dysfunction, and coronary artery disease: Common links. Eur. Urol. 2007, 52, 1590-1600. [CrossRef] [PubMed]

25. Gerber, R.E.; Vita, J.A.; Ganz, P.; Wager, C.G.; Araujo, A.B.; Rosen, R.C.; Kupelian, V. Association of peripheral microvascular dysfunction and erectile dysfunction. J. Urol. 2015, 193, 612-617. [CrossRef] [PubMed]

26. Choo, S.H.; Lee, S.W.; Chae, M.R.; Kang, S.J.; Sung, H.H.; Han, D.H.; Chun, J.N.; Park, J.K.; Kim, C.Y.; Kim, H.K.; et al. Effects of eupatilin on the contractility of corpus cavernosal smooth muscle through nitric oxide-independent pathways. Andrology 2017, 5, 1016-1022. [CrossRef] [PubMed]

27. Chen, C.; Tang, Q.; Zhang, Y.; Yu, M.; Jing, W.; Tian, W. Physioxia: A more effective approach for culturing human adipose-derived stem cells for cell transplantation. Stem Cell Res. Ther. 2018, 9, 148. [CrossRef] [PubMed]

28. Lei, H.; Xin, H.; Guan, R.; Xu, Y.; Li, H.; Tian, W.; Wang, L.; Gao, Z.; Guo, Y.; Lue, T.F.; et al. Low-intensity Pulsed ultrasound improves erectile function in streptozotocin-induced type I diabetic rats. Urology 2015, 86, e11-e18. [CrossRef] [PubMed]

29. Peyton, C.C.; Colaco, M.A.; Kovell, R.C.; Kim, J.H.; Terlecki, R.P. Erectile Dysfunction is Predictive of Endothelial Dysfunction in a Well Visit Population. J. Urol. 2016, 195, 1045-1050. [CrossRef] [PubMed]

30. Liang, X.; Huang, X.; Zhou, Y.; Jin, R.; Li, Q. Mechanical stretching promotes skin tissue regeneration via enhancing mesenchymal stem cell homing and transdifferentiation. Stem Cells Transl. Med. 2016, 5, 960-969. [CrossRef] [PubMed]

31. Jeon, S.H.; Shrestha, K.R.; Kim, R.Y.; Jung, A.R.; Park, Y.H.; Kwon, O.; Kim, G.E.; Kim, S.H.; Kim, K.H.; Lee, J.Y. Combination Therapy Using Human Adipose-derived Stem Cells on the Cavernous Nerve and Low-energy Shockwaves on the Corpus Cavernosum in a Rat Model of Post-prostatectomy Erectile Dysfunction. Urology 2016, 88, e1-e9. [CrossRef] [PubMed]

32. Luo, T.; Liu, H.; Feng, W.; Liu, D.; Du, J.; Sun, J.; Wang, W.; Han, X.; Guo, J.; Amizuka, N.; et al. Adipocytes enhance expression of osteoclast adhesion-related molecules through the CXCL12/CXCR4 signalling pathway. Cell. Prolif. 2017, 50, e12317. [CrossRef] [PubMed]

33. Marquez-Curtis, L.A.; Gul-Uludag, H.; Xu, P.; Chen, J.; Janowska-Wieczorek, A. CXCR4 transfection of cord blood mesenchymal stromal cells with the use of cationic liposome enhances their migration toward stromal cell-derived factor-1. Cytotherapy 2013, 15, 840-849. [CrossRef] [PubMed]

34. Im, G.I.; Kim, H.J.; Lee, J.H. Chondrogenesis of adipose stem cells in a porous PLGA scaffold impregnated with plasmid DNA containing SOX trio (SOX-5, -6 and -9) genes. Biomaterials 2011, 32, 4385-4392. [CrossRef] [PubMed] 
35. De Bock, K.; Georgiadou, M.; Schoors, S.; Kuchnio, A.; Wong, B.W.; Cantelmo, A.R.; Quaegebeur, A.; Ghesquiere, B.; Cauwenberghs, S.; Eelen, G.; et al. Role of PFKFB3-driven glycolysis in vessel sprouting. Cell. 2013, 154, 651-663. [CrossRef] [PubMed]

36. Han, F.; Zhang, S.; Hou, N.; Wang, D.; Sun, X. Irisin improves endothelial function in obese mice through the AMPK-eNOS pathway. Am. J. Physiol. 2015, 309, H1501-H1508. [CrossRef] [PubMed]

37. Lin, C.S.; Xin, Z.C.; Wang, Z.; Deng, C.; Huang, Y.C.; Lin, G.; Lue, T.F. Stem cell therapy for erectile dysfunction: A critical review. Stem Cells Dev. 2012, 21, 343-351. [CrossRef] [PubMed]

38. Xu, W.; Liu, L.Z.; Loizidou, M.; Ahmed, M.; Charles, I.G. The role of nitric oxide in cancer. Cell Res. 2002, 12, 311-320. [CrossRef] [PubMed]

39. Wang, X.; Liu, C.; Li, S.; Xu, Y.; Chen, P.; Liu, Y.; Ding, Q.; Wahafu, W.; Hong, B.; Yang, M. Hypoxia precondition promotes adipose-derived mesenchymal stem cells based repair of diabetic erectile dysfunction via augmenting angiogenesis and neuroprotection. PLoS ONE 2015, 10, e0118951. [CrossRef] [PubMed]

40. Agrawal, H.; Shang, H.; Sattah, A.P.; Yang, N.; Peirce, S.M.; Katz, A.J. Human adipose-derived stromal/stem cells demonstrate short-lived persistence after implantation in both an immunocompetent and an immunocompromised murine model. Stem Cell Res. Ther. 2014, 5, 142. [CrossRef] [PubMed]

41. Liu, G.Y.; Jiang, X.X.; Zhu, X.; He, W.Y.; Kuang, Y.L.; Ren, K.; Lin, Y.; Gou, X. ROS activates JNK-mediated autophagy to counteract apoptosis in mouse mesenchymal stem cells in vitro. Acta Pharmacol. Sin. 2015, 36, 1473-1479. [CrossRef] [PubMed]

42. Lu, Z.Y.; Zhou, H.S.; Su, Z.X.; Qi, J.; Zhang, J.; Xue, G.H.; Li, Y.; Hao, C.N.; Shi, Y.Q.; Duan, J.L. Therapeutic ultrasound plus pulsed electromagnetic field improves recovery from peripheral arterial disease in hypertension. Am. J. Transl. Res. 2017, 9, 4184-4194. [PubMed]

(C) 2018 by the authors. Licensee MDPI, Basel, Switzerland. This article is an open access article distributed under the terms and conditions of the Creative Commons Attribution (CC BY) license (http:/ / creativecommons.org/licenses/by/4.0/). 